\title{
Synchronization of Complex Dynamical Networks with Two Delay Couplings via Intermittent Control
}

\author{
Xiaolong Qi, Huiling Wang, He Zhao, Yi Liang \\ Department of Electronics and Information Engineering, Yili Normal University, Yining 835000, China
}

\begin{abstract}
In this paper, a synchronization scheme for complex delayed dynamical networks with two couplings is investigated via periodically intermittent control methods. The scheme contains system delay and coupling delay, and its coupling matrixes are nonsymmetric. Some exponential synchronization criteria are derived on strict mathematics proofs. And neither dynamical system delay nor coupling delay of the scheme depends on the control width and the non-control width. At last, a numerical simulation shows effectiveness of the proposed scheme.
\end{abstract}

KEYWORD: complex network; exponential synchronization; intermittent control

\section{INTRODUCTION}

In 1998, Pecora and Carroll [1] proposed master stability functi amount of work has been devoted to the study of synchronization in complex networks. Meanwhile, many control methods have been developed to drive complex networks to synchronize such as pinning control [2-8], intermittent control [913 ] and so on.

In some networks circumstance, delayed couplings can be ignored on some links in the complex network, and others caused by traffic congestions and long distance between two nodes are significant, and must be considered. In [12-16], the synchronization in complex networks with both non-delayed and delayed couplings has been investigated. Further, for neural networks with multiple time-varying delays, Zhang investigated its the global asymptotic stability, and obtained less conservative results[17,18].

Intermittent control has been widely used in many fields of application. Yang et al.[9] considered a coupled neural network model with diffusively couplings and stochastic perturbations by utilizing intermittent control, and obtained several sufficient conditions ensuring exponential synchronization. In [10], intermittent control was extended to study a complex network with time-varying discrete delayed coupling. In [11], the exponential synchronization for a class of complex networks with finite distributed delayed coupling is studied via periodically intermittent control, in which the synchronized state is a non-decoupled state. To the best of our knowledge, the existing synchronization schemes only have one delay.

In the paper, periodically intermittent control methods are used to investigate a synchronization scheme for complex delayed dynamical networks with non-delayed and delayed couplings, including system delay and coupling delay, and some exponential synchronization criteria are derived when the coupling matrixes are nonsymmetric. The proposed scheme is more close to the reality and is suitable for a broader range of applications. Moreover, for a class of complex delayed dynamical networks, some sufficient conditions are obtained to ensure global exponential synchronization. A numerical simulation gives effectiveness of the scheme.

\section{PRELIMINARIES}

Consider a complex dynamical network of $N$ identical coupled nodes, which is described by

$\left\{\begin{array}{l}\dot{\boldsymbol{x}}_{i}(t)=\boldsymbol{f}\left(\boldsymbol{x}_{i}(t), \boldsymbol{x}_{i}\left(t-\tau_{0}\right)\right)+c_{0} \sum_{j=1}^{N} a_{i j} \boldsymbol{\Gamma} \boldsymbol{x}_{j}(t) \\ +c_{1} \sum_{j=1}^{N} b_{i j} \boldsymbol{\Gamma} \boldsymbol{x}_{j}\left(t-\tau_{1}\right)+\boldsymbol{u}_{i}(t),(i=1,2, \ldots, N),\end{array}\right.$

where $\boldsymbol{x}_{i}(t)=\left(x_{i 1}(t), x_{i 2}(t), \ldots, x_{i n}(t)\right)^{T} \in \boldsymbol{R}^{n}$ is the state vector of the $i$ th delayed dynamical node, $\boldsymbol{f}: \boldsymbol{R}^{n} \rightarrow \boldsymbol{R}^{n}$ is a continuous vector-valued function, $c_{0}$ and $c_{1}$ are the strength of the non- 
delayed and delayed couplings; $\Gamma \in \boldsymbol{R}^{n \times n}$ is the inner coupling matrix; the delays $\tau_{0}$ and $\tau_{1}$ are bounded constants; $\boldsymbol{A}=\left(a_{i j}\right)_{N \times N}$ and $\boldsymbol{B}=\left(b_{i j}\right)_{N \times N}$ are the non-delay and delay $\tau_{1}$ coupling matrixes, respectively, where $a_{i j}$ and $b_{i j}$ are defined as follows: if there is a non-delayed coupling link from node $i$ to node $j(j \neq i)$, then $a_{i j}>0$; otherwise, $a_{i j}=0$; if there is a delay $\tau_{1}$ coupling link from node $i$ to node $j(j \neq i)$, then $b_{i j}>0$; otherwise, $b_{i j}=0$. In addition, let us assume that $\boldsymbol{A}$ and $\boldsymbol{B}$ are diffusive matrixes, satisfying $\sum_{j=1}^{N} a_{i j}=0$; $\sum_{j=1}^{N} b_{i j}=0(i=1,2, \ldots, N) . \boldsymbol{A}$ and $\boldsymbol{B}$ are seen as two coupling matrixes for sub-networks in one complex network.

$\boldsymbol{u}_{i}(t)=\left\{\begin{array}{l}-d_{i}\left(\boldsymbol{x}_{i}(t)-\boldsymbol{s}(t)\right), t \in[m T,(m+\theta) T), \\ 0, \quad t \in[(m+\theta) T,(m+1) T),\end{array}\right.$

$\theta \quad(0<\theta<1)$ is called the rate of control duration, $T$ is the control period, $\theta T$ is called the control width and $(1-\theta) T$ is called non-control width. $d_{i}$ is a positive constant called control gain, $m \in N^{+}=\{0,1,2, \ldots\}$.

For Eq.(1), its initial conditions are given by $\boldsymbol{x}_{i}(t), t \in[-\tau, 0]$, where $\tau=\max \left(\tau_{0}, \tau_{1}\right)$. Assume that Eq.(1) has a unique solution with respect to initial conditions. Let that $s(t) \in \boldsymbol{R}^{n}$ is a solution of an isolated node, satisfying

$\dot{\boldsymbol{s}}(t)=\boldsymbol{f}\left(\boldsymbol{s}(t), \boldsymbol{s}\left(t-\tau_{0}\right)\right)$.

If $\lim _{t \rightarrow \infty}\left\|\boldsymbol{x}_{i}(t)-\boldsymbol{s}(t)\right\|_{2}=0$, the control dynamical network (1) is said to achieve synchronization.

Define error vector as

$\boldsymbol{e}_{i}(t)=\boldsymbol{x}_{i}(t)-\boldsymbol{s}(t),(i=1,2, \ldots, N)$.

From Eqs. (1), (2), (3) and (4), we can obtain the following error system:

$$
\left\{\begin{array}{l}
\dot{\boldsymbol{e}}_{i}(t)=\boldsymbol{f}\left(\boldsymbol{x}_{i}(t), \boldsymbol{x}_{i}\left(t-\tau_{0}\right)\right)-\boldsymbol{f}\left(s(t), s\left(t-\tau_{0}\right)\right)+ \\
c_{0} \sum_{j=1}^{N} a_{i j} \boldsymbol{\Gamma} \boldsymbol{e}_{j}(t)+c_{1} \sum_{j=1}^{N} b_{i j} \boldsymbol{\Gamma} \boldsymbol{e}_{j}\left(t-\tau_{1}\right)+\boldsymbol{u}_{i}(t), \\
(i=1,2, \ldots, N) .
\end{array}\right.
$$

Next, we present some hypothesises and lemmas for later use.

Hypothesis 1[11] There exist a constant $L_{1}$ and a positive constant $L_{2}$ such that $f$ satisfies the following inequality:
$(\boldsymbol{x}(t)-\boldsymbol{y}(t))^{T}\left[\boldsymbol{f}\left(\boldsymbol{x}(t), \boldsymbol{x}\left(t-\tau_{0}\right)\right)-\boldsymbol{f}\left(\boldsymbol{y}(t), \boldsymbol{y}\left(t-\tau_{0}\right)\right)\right]$

$\leq L_{1}(\boldsymbol{x}(t)-\boldsymbol{y}(t))^{T}(\boldsymbol{x}(t)-\boldsymbol{y}(t))$

$(\boldsymbol{x}(t)-\boldsymbol{y}(t))^{T}\left[\boldsymbol{f}\left(\boldsymbol{x}(t), \boldsymbol{x}\left(t-\tau_{0}\right)\right)-\boldsymbol{f}\left(\boldsymbol{y}(t), \boldsymbol{y}\left(t-\tau_{0}\right)\right)\right]$

$\leq L_{1}(\boldsymbol{x}(t)-\boldsymbol{y}(t))^{T}(\boldsymbol{x}(t)-\boldsymbol{y}(t))+L_{2}\left(\boldsymbol{x}\left(t-\tau_{0}\right)\right.$

$\left.-\boldsymbol{y}\left(t-\tau_{0}\right)\right)^{T}\left(\boldsymbol{x}\left(t-\tau_{0}\right)-\boldsymbol{y}\left(t-\tau_{0}\right)\right)$

for any $\boldsymbol{x}, \boldsymbol{y} \in \boldsymbol{R}^{n}$.

Lemma 1 [19] Let $\boldsymbol{X}$ and $\boldsymbol{Y}$ be arbitrary $n$ dimensional real vectors, $\boldsymbol{K}$ be a positive definite matrix, and $\boldsymbol{P} \in \boldsymbol{R}^{n \times n}$. Then, the following matrix inequality holds:

$$
2 \boldsymbol{X}^{\mathrm{T}} \boldsymbol{P} \boldsymbol{Y} \leq \boldsymbol{X}^{\mathrm{T}} \boldsymbol{P} \boldsymbol{K}^{-1} \boldsymbol{P}^{\mathrm{T}} \boldsymbol{X}+\boldsymbol{Y}^{\mathrm{T}} \boldsymbol{K} \boldsymbol{Y} .
$$

Lemma 2 [20] Let eigenvalues of the matrix $\boldsymbol{A}$ have $\lambda_{1}, \lambda_{2}, \ldots, \lambda_{n}$, and let eigenvalues of the matrix $\boldsymbol{B}$ have $\mu_{1}, \mu_{2}, \ldots, \mu_{m}$, then $n . m$ eigenvalues of the matrix $\boldsymbol{A} \otimes \boldsymbol{B}$ are $\lambda_{i} \mu_{j}(i=1,2, \ldots, n ; j=1,2, \ldots, m)$. where $\otimes$ is Kronecker product.

\section{SYNCHRONIZATION CRITERIA OF \\ COMPLEX DYNAMICAL NETWORKS WITH NON-DELAYED AND DELAYED \\ COUPLINGS VIA INTERMITTENT CONTROL}

In the following, we first give exponential synchronization criterion of the scheme with linear feedback controllers.

Now assume that $\tilde{\boldsymbol{A}}^{s}=\frac{\tilde{\boldsymbol{A}}^{T}+\tilde{\boldsymbol{A}}}{2}$ and $\tilde{\boldsymbol{B}}=\frac{\boldsymbol{B}^{T}+\boldsymbol{B}}{2}$, where $\tilde{A}$ is a modified matrix $\boldsymbol{A}$ via replacing the diagonal elements $a_{i i}$ by $\left(\frac{\rho_{\min }}{\rho}\right) a_{i i}, \rho=\|\Gamma\|$, $\rho_{\min }$ is the minimum eigenvalue of the matrix $\frac{\Gamma^{T}+\Gamma}{2}$. Where $\|\cdot\|$ denotes the Euclidean norm.

Theorem 1 Suppose that Hypothesis 1 holds. If there exist positive constants $\alpha, k$ and $d_{i}$, $(i=1,2, \ldots, l)$,such that

1) $\left(L_{1}+\alpha+\frac{c_{1} k}{2}\right) \boldsymbol{I}_{N}+c_{0} \rho \tilde{\boldsymbol{A}}^{s}-\boldsymbol{D}<\mathbf{0}$,

2) $L_{2}+\gamma<\alpha$

3) $\zeta-2(\alpha+\beta)(1-\theta)>0$,

Where $\boldsymbol{I}_{N}$ is a unit matrix of order $N$, $\boldsymbol{D}=\operatorname{diag}\left(d_{1}, d_{2}, \ldots, d_{N}\right), \quad \beta=\lambda_{\max }\left(\left(L_{1}+\frac{c_{1} k}{2}\right) \boldsymbol{I}_{N}+c_{0} \rho \tilde{\boldsymbol{A}}^{s}\right)$, $\mathrm{c}$, and $\zeta$ is the unique positive solution of the equation $-2 \alpha+\zeta+2\left(L_{2}+\gamma\right) \exp \{\zeta \tau\}=0$. Then the control dynamical network (1) globally exponentially synchronizes. 
First, we show that $\zeta$ is the unique positive solution of the above equation.

Denote $g(\zeta)=-2 \alpha+\zeta+2\left(L_{2}+\gamma\right) \exp \{\zeta \tau\}=0$, Since $L_{2}+\gamma<\alpha, L_{2}>0, \gamma>0$, we obtain

$$
g(0)=-2 \alpha+2\left(L_{2}+\gamma\right)<0, \quad \lim _{\zeta \rightarrow \infty} g(\zeta)=\infty, \text { and }
$$
$g^{\prime}(\zeta)>0$. Hence, $\zeta$ is the unique positive solution of the equation:

$$
-2 \alpha+\zeta+2\left(L_{2}+\gamma\right) \exp \{\zeta \tau\}=0 .
$$

Proof: We assume the candidate Lyapunov function:

$V(t)=\frac{1}{2} \sum_{i=1}^{N} \boldsymbol{e}_{i}^{\mathrm{T}}(t) \boldsymbol{e}_{i}(t)$.

By differentiating the $V(t)$ along the trajectories and using Eqs, using (2), (4), (5) and Hypothesis 1, we can obtain

$$
\begin{aligned}
& t \in {[m T,(m+\theta) T), \quad m \in N^{+}, } \\
& \dot{V}(t)= \sum_{i=1}^{N} \boldsymbol{e}_{i}^{\mathrm{T}}(t) \dot{\boldsymbol{e}}_{i}(t) \\
&= \boldsymbol{e}_{i}^{\mathrm{T}}(t)\left(\boldsymbol { f } \left(\boldsymbol{x}_{i}(t), \boldsymbol{x}_{i}\left(t-\tau_{0}\right)-\boldsymbol{f}\left(s(t), s\left(t-\tau_{0}\right)+\right.\right.\right. \\
&\left.c_{0} \sum_{j=1}^{N} a_{i j} \Gamma \boldsymbol{e}_{j}(t)+c_{1} \sum_{j=1}^{N} b_{i j} \Gamma \boldsymbol{e}_{j}\left(t-\tau_{1}\right)+\boldsymbol{u}_{i}(t)\right) \\
& \leq L_{1} \sum_{i=1}^{N} \boldsymbol{e}_{i}^{\mathrm{T}}(t) \boldsymbol{e}_{i}(t)+L_{2} \sum_{i=1}^{N} \boldsymbol{e}_{i}^{\mathrm{T}}\left(t-\tau_{0}\right) \boldsymbol{e}_{i}\left(t-\tau_{0}\right) \\
&+c_{0} \sum_{i=1}^{N} \sum_{j=1}^{N} a_{i j} \boldsymbol{e}_{i}^{\mathrm{T}}(t) \boldsymbol{\Gamma} \boldsymbol{e}_{j}(t)+c_{0} \sum_{i=1}^{N} \rho_{\min } a_{i i} \boldsymbol{e}_{i}^{\mathrm{T}}(t) \boldsymbol{e}_{i}(t) \\
&+c_{1} \boldsymbol{e}^{T}(t)(\tilde{\boldsymbol{B}} \otimes \Gamma) \boldsymbol{e}\left(t-\tau_{1}\right)-\sum_{i=1}^{l} d_{i} \boldsymbol{e}_{i}^{\mathrm{T}}(t) \boldsymbol{e}_{i}(t) \\
& \leq L_{1} \sum_{i=1}^{N} \boldsymbol{e}_{i}^{\mathrm{T}}(t) \boldsymbol{e}_{i}(t)+L_{2} \sum_{i=1}^{N} \boldsymbol{e}_{i}^{\mathrm{T}}\left(t-\tau_{0}\right) \boldsymbol{e}_{i}\left(t-\tau_{0}\right) \\
&+ c_{0} \rho \sum_{\substack{i=1 \\
i \neq j}}^{N} \sum_{j=1}^{N} a_{i j}\left\|\boldsymbol{e}_{i}^{\mathrm{T}}(t)\right\|\left\|\boldsymbol{e}_{j}(t)\right\|+c_{0} \sum_{i=1}^{N} \rho_{\min } a_{i i} \boldsymbol{e}_{i}^{\mathrm{T}}(t) \boldsymbol{e}_{i}(t) \\
&+c_{1} \boldsymbol{e}^{T}(t)(\tilde{\boldsymbol{B}} \otimes \Gamma) \boldsymbol{e}\left(t-\tau_{1}\right)-\sum_{i=1}^{l} d_{i} \boldsymbol{e}_{i}^{\mathrm{T}}(t) \boldsymbol{e}_{i}(t) .
\end{aligned}
$$

Here $\boldsymbol{e}(t)=\left(\boldsymbol{e}_{1}(t), \boldsymbol{e}_{2}(t), \ldots, \boldsymbol{e}_{N}(t)\right)^{T}$. From Lemma 1 and Lemma 2, we have

$$
\begin{aligned}
& c_{1} \boldsymbol{e}^{T}(t)(\tilde{\boldsymbol{B}} \otimes \Gamma) \boldsymbol{e}\left(t-\tau_{1}\right) \leq \frac{c_{1}}{2 k} \boldsymbol{e}^{T}(t) \boldsymbol{e}(t) \\
& +\frac{c_{1} k}{2} \boldsymbol{e}^{T}\left(t-\tau_{1}\right)(\tilde{\boldsymbol{B}} \otimes \Gamma)^{T}(\tilde{\boldsymbol{B}} \otimes \Gamma) \boldsymbol{e}\left(t-\tau_{1}\right) \\
& =\frac{c_{1}}{2 k} \boldsymbol{e}^{T}(t) \boldsymbol{e}(t)+\frac{c_{1} k}{2} \boldsymbol{e}^{T}\left(t-\tau_{1}\right)\left(\tilde{\boldsymbol{B}}^{T} \tilde{\boldsymbol{B}} \otimes \Gamma^{T} \boldsymbol{\Gamma}\right) \boldsymbol{e}\left(t-\tau_{1}\right)
\end{aligned}
$$

$\dot{V}(t) \leq \boldsymbol{e}^{T}(t)\left[\left(\left(L_{1}+\frac{c_{1}}{2 k}+\alpha\right) \boldsymbol{I}_{N}+c_{0} \rho \tilde{\boldsymbol{A}}^{s}-\boldsymbol{D}\right) \otimes \boldsymbol{I}_{n}\right] \boldsymbol{e}(t)$

$+L_{2} \boldsymbol{e}^{\mathrm{T}}\left(t-\tau_{0}\right) \boldsymbol{e}\left(t-\tau_{0}\right)$

$-\alpha \boldsymbol{e}^{T}(t) \boldsymbol{e}(t)+\frac{c_{1} k}{2} \boldsymbol{e}^{T}\left(t-\tau_{1}\right)\left(\tilde{\boldsymbol{B}}^{T} \tilde{\boldsymbol{B}} \otimes \Gamma^{T} \boldsymbol{\Gamma}\right) \boldsymbol{e}\left(t-\tau_{1}\right)$

$\leq-\alpha \boldsymbol{e}^{T}(t) \boldsymbol{e}(t)+L_{2} \boldsymbol{e}^{\mathrm{T}}\left(t-\tau_{0}\right) \boldsymbol{e}\left(t-\tau_{0}\right)+\gamma \boldsymbol{e}^{T}\left(t-\tau_{1}\right) \boldsymbol{e}\left(t-\tau_{1}\right)$

$=-2 \alpha V(t)+2 L_{2} V\left(t-\tau_{0}\right)+2 \gamma V\left(t-\tau_{1}\right)$.

When $t \in[(m+\theta) T,(m+1) T)$, for $m \in N^{+}$,

$$
\begin{aligned}
& \dot{V}(t) \leq \boldsymbol{e}^{T}(t)\left[\left(\left(L_{1}+\frac{c_{1}}{2 k}\right) \boldsymbol{I}_{N}+c_{0} \rho \tilde{\boldsymbol{A}}^{s}\right) \otimes \boldsymbol{I}_{n}\right] \boldsymbol{e}(t) \\
& +L_{2} \sum_{i=1}^{N} \boldsymbol{e}_{i}^{\mathrm{T}}\left(t-\tau_{0}\right) \boldsymbol{e}_{i}\left(t-\tau_{0}\right) \\
& +\frac{c_{1} k}{2} \boldsymbol{e}^{T}\left(t-\tau_{1}\right)\left(\tilde{\boldsymbol{B}}^{T} \tilde{\boldsymbol{B}} \otimes \Gamma^{T} \boldsymbol{\Gamma}\right) \boldsymbol{e}\left(t-\tau_{1}\right) \\
& \leq 2 \beta V(t)+2 L_{2} V\left(t-\tau_{0}\right)+2 \gamma V\left(t-\tau_{1}\right) .
\end{aligned}
$$

In general,

$\left\{\begin{array}{l}\dot{V}(t) \leq-2 \alpha V(t)+2 L_{2} V\left(t-\tau_{0}\right)+2 \gamma V\left(t-\tau_{1}\right), \\ t \in[m T,(m+\theta) T) .\end{array}\right.$

$\left\{\begin{array}{l}\dot{V}(t) \leq 2 \beta V(t)+2 L_{2} V\left(t-\tau_{0}\right)+2 \gamma V\left(t-\tau_{1}\right), \\ t \in[(m+\theta) T,(m+1) T) .\end{array}\right.$

Let $M=\sup _{-\tau \leq q \leq 0}\{V(q)\} \quad, \quad W(t)=\exp \{\zeta t\} V(t) \quad$, $\tau_{0} \leq \tau_{1}=\tau$

$Q_{1}(t)=W(t)-\varepsilon M, \quad \varepsilon(\varepsilon>1)$ is a positive constant.

Obviously,

$Q_{1}(t)<0$, for $t \in[-\tau, 0]$.

Next, we will prove that

$Q_{1}(t)<0$, for $t \in[0, \theta T]$.

Otherwise, there exists a, $t_{1} \in[0, \theta T)$ such that

$Q_{1}\left(t_{1}\right)=0, \quad \dot{Q}_{1}(t) \geq 0$,

$Q_{1}(t)<0, \quad-\tau \leq t<t_{1}$.

$$
\begin{aligned}
& \dot{Q}_{1}(t)=\zeta \exp \left\{\zeta t_{1}\right\} V\left(t_{1}\right)+\exp \left\{\zeta t_{1}\right\} \dot{V}\left(t_{1}\right) \\
& \quad \leq \zeta W\left(t_{1}\right)+\exp \left\{\zeta t_{1}\right\}\left(-2 \alpha V\left(t_{1}\right)+2 L_{2} V\left(t_{1}-\tau_{0}\right)\right. \\
& \left.\quad+2 \gamma V\left(t_{1}-\tau_{1}\right)\right) \\
& =\zeta W\left(t_{1}\right)-2 \alpha \exp \left\{\zeta t_{1}\right\} V\left(t_{1}\right)+2 L_{2} \exp \left\{\zeta t_{1}\right\} V\left(t_{1}-\tau_{0}\right) \\
& \left.+2 \gamma \exp \left\{\zeta t_{1}\right\} V\left(t_{1}-\tau_{1}\right)\right)
\end{aligned}
$$


Note that $W\left(t_{1}\right)=\varepsilon M$, from Eq. (12)

$W\left(t_{1}-\tau_{0}\right)<\varepsilon M, W\left(t_{1}-\tau_{1}\right)<\varepsilon M$.

$$
\dot{Q}_{1}(t)<\left(-2 \alpha+\zeta+2\left(L_{2}+\gamma\right) \exp \{\zeta \tau\}\right) \varepsilon M=0
$$

$\dot{Q}_{1}(t)<0$. This leads to a contradiction with the Eq. (11). Hence, Eq. (10) holds.

Now, we prove that

$W(t)<\varepsilon M \exp \{2(\alpha+\beta)(t-\theta T)$, for $t \in[\theta T, T]$

Let $P_{1}(t)=W(t)-\varepsilon M \exp \{2(\alpha+\beta)(t-\theta T)\}$.

Otherwise, there exists a $t_{1}^{\prime} \in[\theta T, T)$ such that

$P_{1}\left(t_{1}^{\prime}\right)=0, \quad \dot{P}_{1}\left(t_{1}^{\prime}\right) \geq 0$,

$P_{1}(t)<0,-\tau \leq t<t_{1}^{\prime}$.

$\dot{P}_{1}\left(t_{1}^{\prime}\right)=\zeta \exp \left\{\zeta t_{1}^{\prime}\right\} V\left(t_{1}^{\prime}\right)+\exp \left\{\zeta t_{1}^{\prime}\right\} \dot{V}\left(t_{1}^{\prime}\right)$

$-2(\alpha+\beta) \varepsilon M \exp \left\{2(\alpha+\beta)\left(t_{1}^{\prime}-\theta T\right)\right\}$

$=\zeta W\left(t_{1}^{\prime}\right)-2 \alpha W\left(t_{1}^{\prime}\right)+2 L_{2} \exp \left\{\zeta \tau_{0}\right\} W\left(t_{1}^{\prime}-\tau_{0}\right)$

$+2 \gamma \exp \left\{\zeta \tau_{1}\right\} W\left(t_{1}^{\prime}-\tau_{1}\right)$

$\leq \zeta W\left(t_{1}^{\prime}\right)-2 \alpha W\left(t_{1}^{\prime}\right)+2 L_{2} \exp \{\zeta \tau\} W\left(t_{1}^{\prime}-\tau_{0}\right)$

$+2 \gamma \exp \{\zeta \tau\} W\left(t_{1}^{\prime}-\tau_{1}\right)$

Note:

(1) if $\theta T \leq t_{1}^{\prime}-\tau_{1}<t_{1}^{\prime}$, then $\theta T \leq t_{1}^{\prime}-\tau_{0}<t_{1}^{\prime}$,

FormEq.(15),

$W\left(t_{1}^{\prime}-\tau_{0}\right)<\varepsilon M \exp \left\{2(\alpha+\beta)\left(t_{1}^{\prime}-\theta T\right)\right\}$,

$W\left(t_{1}^{\prime}-\tau_{1}\right)<\varepsilon M \exp \left\{2(\alpha+\beta)\left(t_{1}^{\prime}-\theta T\right)\right\}$.

(2) if $\theta T \leq t_{1}^{\prime}-\tau_{0}<t_{1}^{\prime}$ and $t_{1}^{\prime}-\tau_{1}<\theta T$,

$W\left(t_{1}^{\prime}-\tau_{0}\right)<\varepsilon M \exp \left\{2(\alpha+\beta)\left(t_{1}^{\prime}-\theta T\right)\right\}$,

$W\left(t_{1}^{\prime}-\tau_{1}\right)<\varepsilon M \leq \varepsilon M \exp \left\{2(\alpha+\beta)\left(t_{1}^{\prime}-\theta T\right)\right\}$.

(3) if $t_{1}^{\prime}-\tau_{0}<\theta T$, then $t_{1}^{\prime}-\tau_{1}<\theta T_{1}$,

$W\left(t_{1}^{\prime}-\tau_{0}\right)<\varepsilon M \leq \varepsilon M \exp \left\{2(\alpha+\beta)\left(t_{1}^{\prime}-\theta T\right)\right\}$,

$W\left(t_{1}^{\prime}-\tau_{1}\right)<\varepsilon M \leq \varepsilon M \exp \left\{2(\alpha+\beta)\left(t_{1}^{\prime}-\theta T\right)\right\}$.

Hence,

$\dot{P}_{1}\left(t_{1}^{\prime}\right)<\left(-2 \alpha+\zeta+2\left(L_{2}+\gamma\right) \exp \{\zeta \tau\}\right)$

$\varepsilon M \exp \left\{2(\alpha+\beta)\left(t_{1}^{\prime}-\theta T\right)\right\}=0$

$\dot{P}_{1}\left(t_{1}^{\prime}\right)<0$. This leads to a contradiction with the

Eq. (14). Hence, Eq. (13) holds.

From the above analysis, we can obtain

$W(t)<\varepsilon M \exp \{2(\alpha+\beta)(t-\theta T)\}$, for $t \in[-\tau, T]$.

$W(t)<\varepsilon M \exp \{2(\alpha+\beta)(1-\theta) T\}$, for $t \in[-\tau, T]$.
We further consider the case of $t \in[T, 2 T]$.

We prove that

$Q_{2}(t)=W(t)-\varepsilon M \exp \{2(\alpha+\beta)(1-\theta) T\}<0, \mathrm{i}$. $\mathrm{e} W(t)<\varepsilon M \exp \{2(\alpha+\beta)(1-\theta) T\}$, for $t \in[T, T+\theta T]$. (17)

Otherwise, there exists a $t_{2} \in[T, T+\theta T]$ such that

$Q_{2}\left(t_{2}\right)=0, \dot{Q}_{2}(t) \geq 0$,

$Q_{2}(t)<0,-\tau \leq t<t_{2}$.

$\dot{Q}_{2}\left(t_{2}\right)=\zeta W\left(t_{2}\right)+\exp \left\{\zeta t_{2}\right\} \dot{V}\left(t_{2}\right)$

$\leq \zeta W\left(t_{2}\right)+-2 \alpha W\left(t_{2}\right)+2 L_{2} \exp \{\zeta \tau\} W\left(t_{2}-\tau_{0}\right)$

$+2 \gamma \exp \{\zeta \tau\} W\left(t_{2}-\tau_{1}\right)$

From Eq. (19),

$\dot{Q}_{2}\left(t_{2}\right)<\left(-2 \alpha+\zeta+2\left(L_{2}+\gamma\right) \exp \{\zeta \tau\}\right)$,

$\varepsilon M \exp \{2(\alpha+\beta)(1-\theta) T\}=0$

it contradicts the Eq. (18),so Eq. (17) holds. When $t \in[T+\theta T, 2 T]$, we prove that

$W(t)<\varepsilon M \exp \{2(\alpha+\beta)(t-2 \theta T)\}$

i.e. $P_{2}(t)=W(t)-\varepsilon M \exp \{2(\alpha+\beta)(t-2 \theta T)\}<0$.

Otherwise, $\exists t_{2}^{\prime} \in[T+\theta T, 2 T]$ such that

$P_{2}\left(t_{2}^{\prime}\right)=0, \dot{P}_{2}\left(t_{2}^{\prime}\right) \geq 0$,

$P_{2}\left(t_{2}^{\prime}\right)<0,-\tau \leq t<t_{2}^{\prime}$.

$\dot{P}_{2}\left(t_{2}^{\prime}\right)=\zeta W\left(t_{2}^{\prime}\right)+\exp \left\{\zeta t_{2}^{\prime}\right\} \dot{V}\left(t_{2}^{\prime}\right)$

$-2(\alpha+\beta) \varepsilon M \exp \left\{2(\alpha+\beta)\left(t_{2}^{\prime}-2 \theta T\right)\right\}$

$\leq \zeta W\left(t_{2}^{\prime}\right)-2(\alpha+\beta) \varepsilon M \exp \left\{2(\alpha+\beta)\left(t_{2}^{\prime}-2 \theta T\right)\right\}$

$+2 \beta W\left(t_{2}^{\prime}\right)+2 L_{2} \exp \{\zeta \tau\} W\left(t_{2}^{\prime}-\tau_{0}\right)$

$+2 \gamma \exp \{\zeta \tau\} W\left(t_{2}^{\prime}-\tau_{1}\right)$.

For $t_{2}^{\prime} \leq 2 T$, $\varepsilon M \exp \left\{2(\alpha+\beta)\left(t_{2}^{\prime}-2 \theta T\right)\right\} \leq \varepsilon M \exp \{4(\alpha+\beta)(1-\theta) T\}$.

Obviously,

$$
\begin{aligned}
& W\left(t_{2}^{\prime}-\tau_{0}\right)<\varepsilon M \exp \{2(\alpha+\beta)(1-\theta) T\} \\
& <\varepsilon M \exp \{4(\alpha+\beta)(1-\theta) T\}
\end{aligned}
$$

And

$$
\begin{aligned}
& W\left(t_{2}^{\prime}-\tau_{1}\right)<\varepsilon M \exp \{2(\alpha+\beta)(1-\theta) T\} \\
& <\varepsilon M \exp \{4(\alpha+\beta)(1-\theta) T\} \\
& \dot{P}_{2}\left(t_{2}^{\prime}\right)<\left(-2 \alpha+\zeta+2\left(L_{2}+\gamma\right) \exp \{\zeta \tau\}\right) \\
& \varepsilon M \exp \{4(\alpha+\beta)(1-\theta) T\}=0
\end{aligned}
$$

It contradicts the Eq. (21), so Eq. (20) holds. 
By induction, we can derive the following estimation of $W(t)$ for any integer $m$.

For $m T \leq t<(m+\theta) T)$,

$W(t)<\varepsilon M \exp \{2 m(\alpha+\beta)(1-\theta) T\}$

and for $(m+\theta) T \leq t<(m+1) T$

$W(t)<\varepsilon M \exp \{2(\alpha+\beta)[t-(m+1) \theta T]\}$

For any $t \geq 0$, there exists a nonnegative integer $m^{\prime}$, such that $m^{\prime} T \leq t<\left(m^{\prime}+1\right) T$, we can rewrite Eqs. (23) and (24):

For $\left.m^{\prime} T \leq t<\left(m^{\prime}+\theta\right) T\right)$,

$W(t)<\varepsilon M \exp \left\{2 m^{\prime}(\alpha+\beta)(1-\theta) T\right\}$

$\leq \varepsilon M \exp \{2(\alpha+\beta)(1-\theta) t\}$

and for $\left(m^{\prime}+\theta\right) T \leq t<\left(m^{\prime}+1\right) T$,

$W(t)<\varepsilon M \exp \left\{2(\alpha+\beta)\left[t-\left(m^{\prime}+1\right) \theta T\right]\right\}$

$\leq \varepsilon M \exp \{2(\alpha+\beta)(1-\theta) t\}$

Hence,

$\exp \{\zeta t\} V(t)<\varepsilon M \exp \{2(\alpha+\beta)(1-\theta) t\}$.

Let $\varepsilon \rightarrow 1, V(t) \leq M \exp \{-(\zeta-2(\alpha+\beta)(1-\theta)) t\}$.

So $\zeta-2(\alpha+\beta)(1-\theta)>0$, the conclusion of the theorem 1 holds. The proof is complete.

Next, we analyze the synchronization conditions for the scheme.

Let $\quad \boldsymbol{P}=\left(L_{1}+\alpha+\frac{c_{1} k}{2}\right) \boldsymbol{I}_{N}+c_{0} \rho \tilde{\boldsymbol{A}}^{s}, \quad \boldsymbol{P} \quad$ is a symmetric matrix. Obviously, as long as all $d_{i}$ are sufficient large, $\left(L_{1}+\alpha+\frac{c_{1} k}{2}\right) \boldsymbol{I}_{N}+c_{0} \rho \tilde{\boldsymbol{A}}^{s}-\boldsymbol{D}$ is a negative definite matrix, i.e. $\left(L_{1}+\alpha+\frac{c_{1} k}{2}\right) \boldsymbol{I}_{N}+c_{0} \rho \tilde{\boldsymbol{A}}^{s}-\boldsymbol{D}<\mathbf{0}$ meets condition 1) of Theorem 1. Therefore, the control dynamical network (1) synchronize.

As seen from above assumptions and proof, when $\boldsymbol{A}$ is a symmetric matrix, the controlled dynamical network (1) globally exponentially synchronizes.

\section{NUMERICAL SIMULATION}

In this section, a numerical simulation is given to verify the effectiveness of the proposed synchronization scheme. A scale-free network with 100 nodes is selected as the non-delayed coupling network. The scale-free network is constructed using the Barabási-Albert model with $m_{0}=6$ starting nodes. A nearest-neighbor network with 100 nodes is selected as the delayed coupling network, whose node degree is 4 . The inner coupling matrix $\Gamma$ is a unit matrix of order $n$. Dynamical system on each node is the delayed Chua's circuit [12].

The delayed Chua's circuit is described by

$$
\begin{aligned}
& \dot{\boldsymbol{x}}(t)=\boldsymbol{f}(\boldsymbol{x}(t), \boldsymbol{x}(t-\tau)) \\
& =C \boldsymbol{x}(t)+g_{1}(\boldsymbol{x}(t))+g_{2}(\boldsymbol{x}(t-\tau))
\end{aligned} .
$$

Where $\boldsymbol{x}(t)=\left(x_{1}(t), x_{2}(t), x_{3}(t)\right)^{T} \in \boldsymbol{R}^{3}$,

$$
g_{1}(\boldsymbol{x}(t))=\left(-\frac{\delta}{2}(a-b)\left(\left|x_{1}(t)+1\right|-\left|x_{1}(t)-1\right|\right), 0,0\right)^{T} \in \boldsymbol{R}^{3},
$$$$
g_{2}(\boldsymbol{x}(t-\tau))=\left(0,0, \sigma \partial \sin \left(v x_{1}(t-\tau)\right)\right)^{T} \in \boldsymbol{R}^{3},
$$$$
C=\left(\begin{array}{lll}
-\delta(1+b) & \delta & 0 \\
1 & -1 & 1 \\
0 & -\sigma & \pi
\end{array}\right)
$$

$\delta=10, \quad \sigma=19.53, \quad \pi=0.1636, \quad a=-1.4325$, $b=-0.7831, v=0.5, \partial=0.2$. It is easy to verify that

$$
\begin{aligned}
& \boldsymbol{e}_{i}(t)^{\mathrm{T}}\left[\boldsymbol{f}\left(\boldsymbol{x}(t), \boldsymbol{x}\left(t-\tau_{0}\right)\right)-\boldsymbol{f}\left(\boldsymbol{y}(t), \boldsymbol{y}\left(t-\tau_{0}\right)\right)\right] \\
& \leq \lambda_{\max }\left(\frac{\boldsymbol{C}^{T}+\boldsymbol{C}}{2}+\operatorname{diag}\left(-\delta(a-b), 0, \frac{\sigma \partial v}{2} k^{\prime}\right)\right) \boldsymbol{e}_{i}(t)^{\mathrm{T}} \boldsymbol{e}_{i}(t)
\end{aligned}
$$$$
+\boldsymbol{e}_{i}(t-\tau)^{T} \operatorname{diag}\left(0,0, \frac{\sigma \partial v}{2 k^{\prime}}\right) \boldsymbol{e}_{i}(t-\tau) .
$$

Let $L_{1}=\lambda_{\max }\left(\frac{\boldsymbol{C}^{T}+\boldsymbol{C}}{2}+\operatorname{diag}\left(-\delta(a-b), 0, \frac{\sigma \partial v}{2} k^{\prime}\right)\right)$, $L_{2}=\frac{\sigma \partial v}{2 k^{\prime}}$. By choosing $k^{\prime}=2$, we have $L_{1}=11.83$ and $L_{2}=0.488$. Hence, $f$ satisfies Hypothesis 1 . Select $c_{0}=10, c_{1}=0.1, k=1, \quad \alpha=25$, then $\gamma=\frac{c_{1}}{2 k} \lambda_{\max }\left(\tilde{\boldsymbol{B}}^{T} \tilde{\boldsymbol{B}} \otimes \Gamma^{T} \boldsymbol{\Gamma}\right)=1.953 . \quad \beta=\lambda_{\max }\left(\left(L_{1}+\right.\right.$ $\left.\left.\frac{c_{1} k}{2}\right) \boldsymbol{I}_{N}+c_{0} \rho \tilde{\boldsymbol{A}}^{s}\right)=12.33, \quad \eta=L_{1}+\alpha+\frac{c_{1} k}{2}=51.88$. For $\tau_{0}=0.02, \tau_{1}=0.04(\tau=0.04)$, solution of the equation $\quad-2 \alpha+\zeta+2\left(L_{2}+\gamma\right) \exp \{\zeta \tau\}=0 \quad$ is $\zeta=32.2586$. Computing $\theta>1-\frac{\zeta}{2(\alpha+\beta)}=0.5679$, let $\theta=\frac{\theta T}{T}=\frac{0.12}{0.2}=0.6$. In order to avoid selecting too large $d_{i}$, we adopt adaptive control scheme with adaptive controllers (25). By using Runge-Kutta, we choose initial values: $\boldsymbol{s}(0)=(2,1,3)$, $x_{i}(0)=\left((-1)^{i}(2+0.1 i),(-1)^{i}(1+0.1 i),(-1)^{i}(3+0.1 i)\right)$, $d_{i}(0)=1, \quad \xi_{i}=1$. Figs. 1(a), 1(b), 1(c) show evolution trends of errors. 


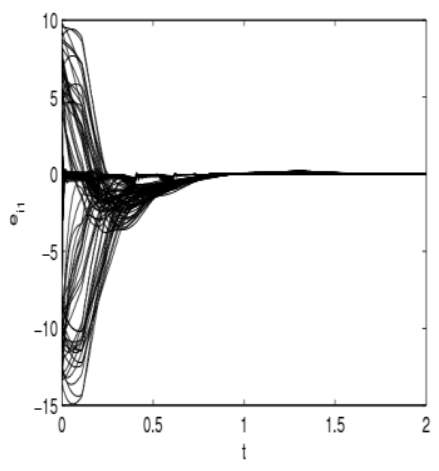

(a)

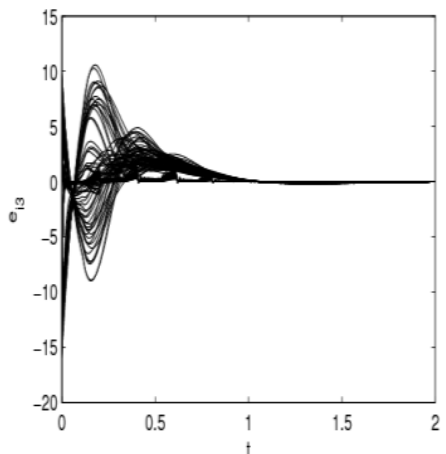

(c)

Fig. 1 Evolution trends of $e_{i 1}, e_{i 2}, e_{i 3}$ of error components, $1 \leq i \leq 100$.

\section{CONCLUSIONS}

In this paper, we introduce the scheme for complex delayed dynamical networks with two couplings via periodically intermittent control. Some exponential synchronization criteria are obtained, and the derived results are less conservative. The scheme is suitable for a broader range of applications. Finally, a numerical simulation shows effectiveness of the scheme.

\section{REFERENCES}

[1] L. M. Pecora, T. L. Carroll, Master stability functions for synchronized coupled systems, Phys.Rev. Lett. 80(1998) 2109-2112.

[2] X. Wang, G. Chen, Pinning control of scale-free dynamical networks, Physica A 310(2002) 521-531.

[3] T. Chen, X. Liu, W. Lu, Pinning complex networks by a single controller, IEEE Trans. Circuits Syst.-I 54(2007) 1317-1326.
[4] M. Porfiri, M. D. Bernardo, Criteria for global pinningcontrollability of complex networks, Automatica 44(2008) 3100-3106.

[5] W. Yu, G. Chen, J. Lü, On pinning synchronization of complex dynamical networks, Automatica 45(2009) 429435.

[6] Q. Song, J. Cao, On pinning synchronization of directed and undirected complex dynamical networks, IEEE Trans. Circ. Syst.-I 57(2010) 672-680.

[7] D. Gong, H. Zhang, Z. Wang, B. Huang, Pinning synchronization for a general complex networks with multiple time-varying coupling delays, Neural Process Lett 35(2012) 221-231.

[8] W. Yu, G. Chen, J. Lü, J. Kurths, Synchronization via pinning control on general complex networks, SIAM J.Control Optim., 51(2013) 1395-1416.

[9] X. Yang, J. Cao, Stochastic synchronization of coupled neural networks with intermittent control, Phys. Lett. A 373 (2009) 3259-3272.

[10] S. Cai, Z. Liu, F. Xu, J. Shen, Periodically intermittent controlling complex dynamical networks with timevarying delays to a desired orbit, Phys. Lett. A 373(2009) 3846-3854.

[11] C. Hu, J. Yu, H. Jiang, Z. Teng, Exponential synchronization of complex networks with finite distributed delays coupling, IEEE Trans. Neural Netw. 12(2011) 1999-2010.

[12] W. Sun, S. Chen, W. Guo, Adaptive global synchronization of a general complex dynamical network with non-delayed and delayed coupling, Phys. Lett. A 372 (2008) 6340-6346.

[13] H. Li, New criteria for synchronization stability of continuous complex dynamical networks with nondelayed and delayed coupling, Commun Nonlinear Sci Numer Simulat 16 (2011) 1027-043.

[14] J. Wang, H. Wu, Local and global exponential output synchronization of complex delayed dynamical networks, Nonlinear Dyn 67(2012) 497-504.

[15] W. Guo, F. Austin, S. Chen, Global synchronization of nonlinearly coupled complex networks with non-delayed and delayed coupling, Commun Nonlinear Sci Numer Simulat 15 (2010) 1631-1639.

[16] X. Wu, H. Lu, Hybrid synchronization of the general delayed and non-delayed complex dynamical networks via pinning control, Neurocomputing 89 (2012) 168-177.

[17] H. Zhang, Z. Wang, D. Liu, Global asymptotic stability of recurrent neural networks with multiple time-varying delays, IEEE Trans. Neural Netw. 19(2008)855-873.

[18] J. Fu, H. Zhang, T. Ma, Q. Zhang, On passivity analysis for stochastic neural networks with interval time-varying delay, Neurocomputing, 73(2010) 795-801.

[19] J. Wu, L. Jiao, Synchronization in complex delayed dynamical networks with nonsymmetric coupling, Physica A 386 (2007) 513-530.

[20] J. Zhou, T. Chen, Synchronization in general complex delayed dynamical networks, IEEE Trans. Circuits Syst. I 53 (2006)733-744. 